\title{
Comparison of outcomes between intravitreal ranibizumab and intravitreal aflibercept for diabetic macular edema with "Treat-and-Extend" regimen
}

Masahiko Sugimoto ( $\nabla$ sugmochi@aqua.ocn.ne.jp )

Mie University School of Medicine https://orcid.org/0000-0001-5147-4183

Shinichiro Chujo

Department of Ophthalmology, Mie University School of Medicine

Taku Sasaki

Department of Ophthalmology, Mie University, School of Medicine

Atsushi Ichio

Department of Ophthalmology, Mie University, School of Medicine

Ryohei Miyata

Department of Ophthalmology, Mie University, School of Medicine

Hisashi Matsubara

Department of Ophthalmology, Mie University, School of Medicine

Mineo Kondo

Department of Ophthalmology, Mie University, School of Medicine

\section{Research article}

Keywords: aflibercept, diabetic macular edema, ranibizumab, treat-and-extend regimen

Posted Date: January 17th, 2020

DOI: https://doi.org/10.21203/rs.2.21161/v1

License: (c) (i) This work is licensed under a Creative Commons Attribution 4.0 International License.

Read Full License 


\section{Abstract}

Background To compare the effectiveness of intravitreal ranibizumab (IVR) and intravitreal aflibercept (IVA) performed with the Treat-and-Extend (TAE) regimen on eyes with diabetic macular edema (DME).

Patients and methods This was a retrospective study of 125 eyes of 125 treatment naïve DME patients who received anti-VEGF injections at 3 consecutive monthly intervals as the loading phase. Of these 125 eyes, 26 eyes completed the treatment with the TAE regimen for at least 24 months. Among the 26 eyes, 13 eyes of 13 patients (mean age, $70.9 \pm 6.0$ years) received intravitreal injections of $0.5 \mathrm{mg}$ ranibizumab and 13 eyes of 13 patients (65.9 \pm 8.6 years) received $2 \mathrm{mg}$ aflibercept. The changes in the best-corrected visual acuity (BCVA), central retinal thickness (CRT), diabetic retinopathy severity (DRS), and total injection numbers were compared between the two anti-VEGF agents.

Results No significant differences were detected in the baseline demographics. At 24 months, the BCVA was significantly improved in both groups; from $0.31 \pm 0.19$ to $0.10 \pm 0.12 \log M A R$ units for IVR and $0.41 \pm 0.19$ to $0.16 \pm 0.28$ logMAR units for IVA ( $P=1.29 \times 10-9)$. The CRT was significantly reduced in both groups; $440.9 \pm 69.3$ to $307.5 \pm 66.4 \mu \mathrm{m}$ for IVR and $473.9 \pm 71.5$ to $317.8 \pm 71.2 \mu \mathrm{m}$ for IVA ( $P=3.55 \times 10-9)$. No significant differences were detected in the improvements of the BCVA and the CRT in both groups, and the total injection numbers were significant fewer for the IVA group $(11.0 \pm 1.2)$ than the IVR group (12.0 \pm 1.0$)$. The DRS was significantly improved in both groups $(P=0.0004$ for IVR and $P=0.009$ for IVA).

Conclusion No significant differences were detected in the improvements of the BCVA or CRT and injection numbers between IVR and IVA groups treated with the TAE regimen. These results indicate that the results of the treatment with both agents with the TAE regimen were equally effective.

\section{Background}

Diabetic macular edema (DME) is a leading cause of blindness in working age individuals [1,2], and many factors are related to its development. It has been determined that the level of vascular endothelial growth factor (VEGF) is associated with the degree of vascular proliferations and hyperpermeability, and its suppression is effective in resolving DME. Many randomized controlled trials have shown that intravitreal anti-VEGF injections is an effective first-line treatment for DME [3-7]. Although the number of injections was reduced over the years, the results of randomized controlled trials have shown that it required repeat injections or frequent visits. Therefore, other flexible regimens are often used, and because of the risk-benefit balance, the optimal regimen has not been definitively established.

Treatment as needed or pro re nata (PRN) regimen is frequently used. The timing of the injection is mainly determined by a criterion, such as the retinal thickness, which is determined from the optical coherence tomographic (OCT) images $[8,9]$ or the best-corrected visual acuity (BCVA). It has been reported that patients required fewer injections with the PRN regimen than the monthly injection regimen. The PRN regimen has also been used for other retinal diseases including age-related macular degeneration (AMD). The results of earlier studies have shown that the PRN regimen for AMD had similar 
visual outcomes as the monthly injection regimen, and comparable outcomes could be achieved with fewer injections [10-14]. In fact, 75.0\% of Japanese ophthalmologists prefer the PRN regimen for DME during the maintenance phase [15]. However, the PRN regimen is inconvenient because it requires frequent visits to determine the status of the DME. In addition, the HORIZON trial found that nonadherence to the monthly monitoring led to a loss of the benefits of the previous treatments [16].

Much attention has been recently given to the Treat-and-Extend (TAE) regimen, and its main goal was to minimize the number of injections and patient visits. The TAE regimen consisted of a loading phase and a maintenance phase in which the injection interval is determined by the disease activity. Many studies have reported on the effectiveness of the TAE regimen for AMD [17-21]. A meta-analysis of 26,360 patients from 42 real-world observational studies of intravitreal ranibizumab (IVR) showed that the TAE regimen resulted in better visual outcomes with fewer visits compared to the PRN regimen for AMD at 2 years [22]. These results indicated that an individualized treatment and follow-up schedule is possible for each patient with the TAE regimen. Relevant to this study, the TAE regimen has also been reported to be effective for DME [23-25]. However, there has been only one small case series study that reported similar BCVAs after an OCT-guided TAE protocol and a visual acuity-guided PRN regimen [26]. So, it is still difficult to define the best treatment regimen for DME.

At present, three anti-VEGF agents, bevacizumab (Avastin, Genentech, South San Francisco, CA, USA ), ranibizumab (Lucentis, Genentech), and aflibercept (Eylea, Regeneron Pharmaceuticals, Tarrytown, NY, USA) have been used to treat DME, and the former two agents have been approved to treat eyes with DME in Japan. However, it had not been definitively determined whether there are differences in the effectiveness in ranibizumab and aflibercept in treating DME. The Diabetic Retinopathy Clinical Research Network (DRCR.net) protocol-T study reported that aflibercept was more effective especially in patients with initially poor vision as long as a strict PRN regimen was followed [27]. However, there is no study that compared the effectiveness of IVR and intravitreal aflibercept (IVA) using the TAE regimen in eyes with DME. Thus, the purpose of this study was to compare the effectiveness of IVR to that of IVA applied with the TAE regimen. and to show its effectiveness with both anti-VEGF agents.

\section{Patients And Method}

This was a retrospective, single-center, comparative case series study. The medical charts of 125 eyes of 125 treatment-naïve DME patients in the database of the Mie University Hospital were examined. The patients had been examined between April 2014 to November 2018. Of the 125 eyes, 55 eyes received IVR and 70 eyes received IVA. The patients received 3 consecutive IVR or IVA injections as a loading phase. Among the 58 eyes of 58 patients, 28 eyes received IVR and 30 eyes received IVA and were treated with the TAE regimen. In the end, 13 eyes of 13 patients who received IVR and 13 eyes of 13 patients who received IVA completed the 24 month treatment with the TAE regimen (Fig. 1).

The procedures used in this study were approved by the Institutional Ethics Review Board of the Mie University Hospital (\#702), and the study was registered at http://www.umin.ac.jp (UMIN ID 000033728). 
The procedures adhered to the tenets of the Declaration of Helsinki.

Each patient had a comprehensive ophthalmological examination including measurements of the bestcorrected visual acuity (BCVA) and intraocular pressure, examination of the anterior segment by slit-lamp biomicroscopy, examination of the fundus by indirect ophthalmoscopy to grade the degree of diabetic retinopathy severity (DRS), and examination by spectral-domain optical coherence tomography (SD-OCT) to determine the macular structure.

The study inclusion criteria were;1) presence of center-involved DME diagnosed by the clinical findings and fluorescein angiography, and a CRT > $250 \mu \mathrm{m}$ in the SD-OCT images at study entry, 2) age at least 20 years, and 3) BCVA pretreatment of $\geq 20 / 320$. The exclusion criteria were; prior ocular surgery including cataract surgery within 6 months and during the experimental period, macular laser photocoagulation, and intravitreal or sub-tenon injections of steroids within 3 months of beginning the study. In addition, eyes with ocular inflammation, drusen, severe proliferative diabetic retinopathy, retinal hemorrhage which involved the intra- or subfoveal spaces, an epiretinal membrane, history of pars plana vitrectomy, glaucoma, and media opacities which affected the OCT imaging, i.e., vitreous hemorrhage, vitreous opacity, severe cataract and corneal opacity, were excluded. Patients with uncontrolled systemic medical conditions or history of thromboembolic events were also excluded. The diabetes control was evaluated by the $\mathrm{HbA} 1 \mathrm{c}$ levels (normal range:4.6-6.2\%), and renal dysfunction was evaluated by the estimated glomerular filtration rate (eGFR; normal range: $60-120 \mathrm{ml} / \mathrm{min} / \mathrm{m}^{2}$ ).

After 3 consecutive monthly IVR or IVA injections as the loading phase, patients who were resistant to the anti-VEGF treatment or could not maintain the financial burden were excluded. They were switched to other treatments including steroid or vitrectomy. Patients who responded to IVR or IVA but did not care for the TAE regimen were switched to the PRN regimen and excluded from the study. Patients who did not complete the 2 year TAE follow-up were also excluded from the statistical analyses.

Intravitreal injection of anti-VEGF agents

Intravitreal anti-VEGF injections were performed under local subconjunctival injection or topical anesthesia. Each patient received $0.5 \mathrm{mg}$ of ranibizumab (IVR group) or $2 \mathrm{mg}$ of aflibercept (IVA group) intravitreally with a 30-gauge needle that was inserted $4 \mathrm{~mm}$ posterior to the corneal limbus under sterile conditions. All patients received topical levofloxacin hydrate, (1.5\% Cravit ophthalmic solution) for 1 week after the injection.

Modified TAE regimen for DME

All patients were given 3 consecutive monthly injections of the IVR or IVA, and they were treated during the maintenance phase with a modified TAE regimen. The follow-up injection intervals were applied as shown in Fig. 2. The first post-loading phase injection interval was 8 weeks, then the injection interval was determined by the TAE protocol. The interval was extended by 2 weeks if the CRT was $<350 \mu \mathrm{m}$ at 2 
consecutive examinations, or the injection interval was shortened by 2 weeks if the CRT was $\geq 350 \mu \mathrm{m}$ or it increased by $>20 \%$ of the baseline value. The minimum injection interval was set at 8 weeks.

Measurements of best-corrected visual acuity (BCVA)

The BCVA was measured with a Landolt chart at every visit. The decimal BCVA was converted to the logarithm of the minimum angle of resolution (logMAR) units for the statistical analyses.

Optical coherence tomography (OCT)

The measurements of the CRT were made on the images obtained by a Heidelberg Spectralis OCT instrument (Heidelberg Engineering Inc, Heidelberg, Germany). For qualitative and quantitative analyses of the OCT images, the fast macula protocol was used to obtain the images with an automatic real time mean value of 9 which acquired 25 horizontal lines consisting of 1024 A-scans per line. The CRT was defined as the thickness between the internal limiting membrane and the retinal pigment epithelium at the fovea, and the value was automatically calculated from the center subfield of the macular thickness map using the bundled software.

Statistical Analyses

The results are presented as the means \pm standard deviations (SDs). Because the sample size was small and the statistical power is low, the Kolmogorov-Smirnov tests were used to determine the significance of the differences between corresponding pairs in the two groups. Two-way repeated measures ANOVA and post-hoc $t$ tests with Bonferroni's corrections were used to determine the significance of the changes in the BCVA and CRT. Chi-squared analysis was used to determine the significance of the differences of the DRS between baseline and 24 months. Two-tailed $P$ values of $<0.05$ were considered to be significant. The statistical evaluations were performed by Statcel 4 Statistical Program (Statcel; OMC, Saitama, Japan).

\section{Results}

\section{Clinical characteristics of patients at baseline (Table 1)}

The clinical characteristics of the patients are summarized in Table 1. Thirteen eyes of 13 DME patients received IVR, and 13 eyes of 13 DME patients received IVA. No significant differences in the baseline values were found between the two groups; the mean age of the IVR group was $70.9 \pm 6.0$ years and that for the IVA group II was $65.9 \pm 8.6$ years $(P=0.08)$, the HbA1c level was $7.6 \pm 1.6 \%$ for the IVR group and $7.4 \pm 1.8 \%$ for the IVA group $(P=0.33)$, and the eGFR level was $87.1 \pm 16.1 \mathrm{ml} / \mathrm{min} / \mathrm{m}^{2}$ for the IVR group and $71.0 \pm 21.7 \mathrm{ml} / \mathrm{min} / \mathrm{m}^{2}$ for the IVA group $(P=0.31)$. The mean baseline BCVA were $0.31 \pm 0.19$ $\log M A R$ units in the IVR group and $0.41 \pm 0.19 \log M A R$ units for the IVA group $(P=0.12$, Table 1$)$. The mean baseline CRT was $440.9 \pm 69.3 \mu \mathrm{m}$ for the IVR group and $473.9 \pm 71.5 \mu \mathrm{m}$ for the IVA group $(P=$ 0.33 , Table 1). 
There were no ocular complications including intraocular pressure elevations, infections, or adverse systemic events that developed during the course of the experimental period for these 26 eyes.

\section{Changes in DRS during TAE treatment regimen (Table 2)}

The results of the fundus examinations classified by the DRS are shown in Table 2. For the IVR group, the number of eyes classified as minimal non-proliferative diabetic retinopathy (NPDR) was 2, moderate NPDR was 8, and severe NPDR was 3 . After 24 months of treatment, the DRS was significantly improved (Chi-squared analysis, $P=0.0004$ ); the number of minimal NPDR was 12 , moderate NPDR was 1 , and severe NPDR was 0.

For the IVA group, the number of minimal NPDR was 5, moderate NPDR was 2, and severe NPDR was 6. After 24 months of treatment, the DRS was significantly improved (Chi-squared analysis, $P=0.009$ ); the number of eyes with minimal NPDR was 12, moderate NPDR was 1, and severe NPDR was 0.

\section{Changes in BCVA and CRT following Treat-and-Extend regimen (Figure 3)}

The BCVA was significantly improved during the observation period $\left(P=1.29 \times 10^{-9}\right.$, Figure 3a). The BCVA was significantly improved to $0.13 \pm 0.16 \operatorname{logMAR}$ units in the IVR group and to $0.24 \pm 0.22 \log M A R$ units in the IVA group after the loading phase. At 6 months, the BCVA was improved to $0.13 \pm 0.11 \log M A R$ units in the IVR group and $0.18 \pm 0.18 \operatorname{logMAR}$ units in the IVA group, and at 12 months, the BCVA was $0.14 \pm 0.18 \log M A R$ units in the IVR group I and $0.14 \pm 0.17 \log M A R$ units in the IVA group II. At 18 months, the BCVA was $0.10 \pm 0.17 \log M A R$ units in the IVR group and $0.17 \pm 0.31 \log M A R$ units in the IVA group. The final BCVA at 24 months was significantly improved to $0.10 \pm 0.12 \log M A R$ units in the IVR group and $0.16 \pm 0.28 \operatorname{logMAR}$ units in the IVA group. No significant differences were observed between the groups at all times $(P>0.05)$.

The CRT was also significantly improved during the observation period ( $P=3.55 \times 10^{-9}$,Figure 3b); 334.7 $\pm 99.3 \mu \mathrm{m}$ for the IVR group and $348.7 \pm 53.5 \mu \mathrm{m}$ in the IVA group after the loading phase, $352.2 \pm 95.1$ $\mu \mathrm{m}$ in the IVR group and $333.09 \pm 82.0 \mu \mathrm{m}$ in the IVA group at 6 months, $311.8 \pm 60.9 \mu \mathrm{m}$ in the IVR group and $332.9 \pm 72.6 \mu \mathrm{m}$ in the IVA group at 12 months, $310.2 \pm 58.2 \mu \mathrm{m}$ in the IVR group and $299.9 \pm 62.6$ $\mu \mathrm{m}$ in the IVA group at 18 months. The mean final CRT at 24 months was significantly improved to 307.5 $\pm 66.4 \mu \mathrm{m}$ in the IVR group and $317.8 \pm 71.2 \mu \mathrm{m}$ in the IVA group. No significant differences were observed between the groups at all times $(P>0.05)$.

\section{Number of injections with TAE regimen (Figure 4 and Table 3)}


The mean number of injections at 12 months was $7.1 \pm 0.3$ in the IVR group (range 7 to 8 ) and $6.5 \pm 0.5$ in the IVA group (range 6 to $7 ; P=0.13$, Kolmogorov-Smirnov tests, Figure 4a and Table 3). The mean injection number at 18 months was $9.5 \pm 0.8$ in the IVR group (range 9 to 11) and $8.8 \pm 0.9$ in the Iva group (range 8 to $11, P=0.13$, Figure $4 \mathrm{~b}$ and Table 3 ). The mean number of injections at 24 months was $12.0 \pm 1.0$ in the IVR group (range 11 to 14 ) and $11.0 \pm 1.2$ in the IVA group (range 10 to $14, P=0.29$, Figure $4 \mathrm{c}$ and Table 3). The mean interval between injections at 24 months was $12.0 \pm 3.4$ weeks in the IVR group and $12.2 \pm 3.2$ weeks in the IVA group $(P=0.90)$. The mean maximum interval was 16 weeks in the IVR group and 16 weeks in the IVA group.

\section{Discussion}

Our results showed that there were no significant differences in the BCVA, CRT, number of injections/2 years, and intervals between IVR and IVA injections applied with the TAE regimen in eyes with DME.

Many earlier studies have reported improvements of the DR after multiple injections of anti-VEGF agents. The subgroup analyses of the RIDE \& RISE and the VIVID \& VISTA studies showed improvements of the DR in eyes with DME. These results showed the possibility that anti-VEGF agents can replace photocoagulation as a DR treatment [28-31]. The Phase 3, double-masked, randomized PANORAMA study was designed to test the efficacy and safety of IVA in patients with moderately severe to severe NPDR compared to sham injections (ClinicalTrials.gov Identifier: NCT02718326). The study reported that the proportion of patients with $\geq 2$-step improvement in the severity of the DR was significantly higher after aflibercept than after the sham injection (presented at Bascom Palmer Eye Institute's Angiogenesis, Exudation, and Degeneration 2019 symposium in Miami, Feb 9, 2019, Miami). Such improvements occurred due to the biological aspects of the anti-VEGF agents on the structural vascular features and the alterations of the hemodynamic properties [32]. Although these studies mentioned the effectiveness of anti-VEGF agents for DR improvement, they required many injections to obtain improvement. For example, Protocol-S required a mean of 14 injections (range 10 to 17) for a 2 year study period for eyes with DME at the baseline [28]. We also found an improvement of the DR after 2-year treatment with the TAE regimen, and our injection numbers were fewer than that of other studies (12.0 \pm 1.0 for the IVR group and $11.0 \pm 1.2$ for the IVA group). These differences indicate the possibility that DR improvement can be obtained with fewer injections with both IVR and IVA applied with the TAE regimen. Thus, the TAE regimen should be considered to be a good option for DR treatment.

For eyes with $A M D$, the TAE regimen required more injections than the PRN regimen but required fewer visits with better visual outcomes [22]. When we compared our results to that of previous two-year studies on DME including those using the TAE regimen, e.g., the RETAIN study and T-REX DME, our results are comparable to them even though the patient background and treatment protocol differed (Table 4). The changes in the mean BCVA and CRT with the TAE regimen appear to be comparable to that with the PRN regimen as reported by the DRCR.net protocol-T. A fixed injection appears to have better improvements but it required more injections than with the PRN or the TAE regimens. On the other hand, the injection numbers were fewer with PRN but the intervals were longer with the TAE regimen. The RETAIN study [23] 
compared the outcomes of the IVR-TAE regimen with the IVR-PRN regimen, and the results showed that the injection intervals could be extended to more than 8 weeks for $70 \%$ of the patients $(9.2$ weeks for TAE vs 11.2 weeks for PRN). The T-REX DME study [25] compared the monthly IVR treatment group, IVR-TAE group, and the IVR-TAE combined with laser group. The results of the T-REX study showed that significantly fewer injections were required for both the TAE group than the monthly group (18.9 for TAE vs 24.7 for monthly). In our study using the TAE regimen, the injection interval during the 24 months treatment period was longer than previous studies, viz., $12.0 \pm 3.4$ weeks for IVR and $12.2 \pm 3.2$ weeks for IVA, and the maximum interval was 16.0 weeks for both agents with no significant differences. The intervals were longer than that reported by the RETAIN study (9.2 weeks for IVR) and TREX-DME (6.2 weeks for IVR). The results of earlier studies (Table 4) support our results which indicates the effectiveness of the TAE regimen for DME compared to the as needed PRN regimen.

There are many differences in the re-treatment criterion among the different studies performed on eyes treated with the TAE regimen, i.e., the inactivity criteria, the extension length, and the strategy. Our study was a retrospective study as opposed to the prospective RETAIN and TREX studies. Therefore, it was difficult to compare our results with these other studies. In addition, our TAE regimen used the CRT as the only inactivity criterion. The resolution of intraretinal and subretinal fluids was not included in the criteria in our TAE regimen as with AMD management. In addition, some studies reported no advantage of the TAE regimen compared to monthly or PRN regimens although these were results from a small number of patients and the duration of the studies was only 1-year. Ebneter et al. compared OCT-guided TAE to the visual acuity-guided PRN regimen and found similar visual acuity outcome [26]. Ehlers et al. reported similar visual acuity outcomes for the monthly and TAE regimen for persistent DME and switching from bevacizumab to ranibizumab [33]. There are disadvantages of the TAE regimen [34]; 1) it requires frequent injections in the first year which may result in over treatment, 2) it is difficult to identify a stable status during the treatment, 3 ) there is an increased chance of adverse complications, 4) there is limited evidence for its effectiveness, 6 ) there is no stop criteria, and 7) not all patients can complete the longitudinal treatment protocol [24]. Our result showed that only 26 of the 58 eyes could complete the 2 years of the TAE protocol. These disadvantages indicate the difficulty in completing the TAE regimen as a maintenance phase in the real world. We need more considerations for these disadvantages although our results showed the effectiveness of the both IVR and IVA with the TAE regimen. However, there is a possibility that once the protocol is properly fine-tuned, the TAE regimen may become a good treatment protocol for eyes with DME.

The DRCR.net protocol-T [27] reported that IVA was more effective for eyes with poor baseline BCVA at one year but the difference was not significant at 2 years. There are several factors that can explain the differences between the two agents. Experiments on animals have shown that aflibercept penetrates deeper into the retina than ranibizumab after an intravitreal injection in monkey eyes [35], and the VEGF concentration in the aqueous was suppressed longer after IVA than IVR in the monkey eyes [36]. In addition, aflibercept binds not only to VEGF and placenta growth factor but to galectin-1 [37]. From these different molecular characteristics of the two agents, there may exist different response of the TAE regimen between IVR and IVA. Although the RETAIN and T-REX DME studies reported the usefulness of 
the TAE regimen for DME, none of the earlier studies has shown any differences between IVR and IVA with the TAE regimen. We also could not find any significant differences in the BCVA, CRT, and the injection numbers between IVR and IVA. Similar to our result, Schwarzer et al. reported no significant differences in outcomes between IVR or IVA using OCT-guided TAE protocol without a fixed loading phase [38]. There are some explanations for these differences. First, we did not divide the patients by the BCVA at the baseline which is different from the DRCR.net protocol-T. Second, the DRCR.net protocol-T reported significant differences only at 12 months and no significant difference at 24 months. Therefore, the molecular characteristics of the two agents may be subtle and may not be enough to cause differences as long as patients were treated with the TAE regimen. There is also the possibility that our results were from a small number of participants, and it did not have enough power to show any difference between the agents.

There are some limitations in our study including the small sample size. In addition, we selected a CRT of $350 \mu \mathrm{m}$ as the main criterion to extend the interval between injections. This is comparable to the TREXDME criterion of $325 \mu \mathrm{m}$. However, the RETAIN study based the extension criterion on the visual acuity. It is known that a thinner CRT does not necessarily indicate a better BCVA because there are some cases whose outer segments of the photoreceptors were already damaged before the DME was resolved. This is called a paradoxical change between BCVA and CRT for DME [39]. Because we defined the CRT as a standard for treatment extension, this TAE regimen cannot cover those patients with lower CRT with poorer BCVA. In addition, another study has reported a similar visual acuity outcome between OCT guided-TAE protocol and visual acuity guided-PRN regimen. [26]. Thus, it is still not clear which is the better criterion to use.

Another limitation was that 6 of the 26 eyes of both groups (23.1\%) could not extend the interval to more than 8 weeks. In the RETAIN study, about $30 \%$ patients could not extend the interval to more than 8 weeks. These eyes received fixed 8 weeks injections, and this is not a precise TAE regimen because it contributed less to patients' benefit. It is also important consider other treatment including sustained steroid agents [40-42] for these cases when the injection interval cannot be extended. Finally, we did not compare our TAE results with the PRN regimen. Although the RETAIN study and T-REX DME reported a superiority of the TAE regimen compared to the PRN regimen, it is difficult to argue against the usefulness of PRN regimen. In the real world, we perform extra injections mainly on the complaints of the patients without a strict $P R N$ regimen. So, it is not clear whether a strict PRN regimen works better than TAE regimen, and we need to compare them in the future.

\section{Conclusions}

No significant difference was observed between IVR and IVA with respect to their effectiveness. The TAE regimen with both agents for DME is a good option for patients with DME because it will result in good improvements of the BCVA and CRT.

\section{List Of Abbreviations}


Age-related macular degeneration (AMD), best-corrected visual acuity (BCVA), central retina thickness (CRT), diabetic macular edema (DME), intravitreal aflibercept (IVA), intravitreal ranibizumab (IVR), optical coherence tomography (OCT), pro re nata (PRN), spectral-domain optical coherence tomography (SD$\mathrm{OCT}$ ), standard deviations (SDs), treat-and-Extend (TAE)vascular endothelial growth factor (VEGF).

\section{Declarations}

\section{Ethics approval and consent to participate}

This research was conducted ethically in accordance with the World Medical Association Declaration of Helsinki. The procedures used in this study were approved by the Institutional Ethics Review Board of the Mie University Hospital (\#702), and the study was registered at http://www.umin.ac.jp (UMIN ID 000033728).

\section{Consent for publication}

Not applicable.

\section{Availability of data and materials}

The datasets used and/or analyzed during the current study are available from the corresponding author on reasonable request.

\section{Competing interests}

Masahiko Sugimoto have:

[Financial Support] Alcon Pharma and Bayer.

[Other (lecture fee)] Alcon pharma, Kowa Pharma, Senjyu Pharma, Daiichi Yakuhin Sangyo, Bayer, Wakamoto Pharma.

\section{Funding}

Authors receive no funding of any research relevant to the study.

\section{Authors' contributions}

M.S., and M.K. designed the study. M.S. and S.C. contributed to writing the main manuscript text. S. C., T. S., A.I., R.M. and H.M. recruit patients and analyzed data. All authors reviewed the manuscript.

\section{Acknowledgements}

We thank Professor Emeritus Duco Hamasaki of the Bascom Palmer Eye Institute of the University of Miami for critical discussion and final manuscript revisions. 


\section{Disclosure Statement}

Masahiko Sugimoto have:

[Financial Support] Alcon Pharma and Bayer.

[Other (lecture fee)] Alcon pharma, Kowa Pharma, Senjyu Pharma, Daiichi Yakuhin Sangyo, Bayer, Wakamoto Pharma.

\section{References}

1. McMeel JW, Trempe CL, Franks EB. Diabetic maculopathy. Trans Sect Ophthalmol Am Acad Ophthalmol Otolaryngol. 1977; 83: 476-487.

2. Moss SE, Klein R, Klein BEK. The incidence of visual loss in a diabetic population. Ophthalmology 1988; 95:1340-1348.

3. Haritoglou C, Kook D, Neubauer A, et al. Intravitreal bevacizumab (Avastin) therapy for persistent diffuse diabetic macular edema. Retina 2006; 26:999-1005.

4. Arevalo JF, Fromow-Guerra J, et al. Primary intravitreal bevacizumab (Avastin) for diabetic macular edema. Ophthalmology 2007; 114:743-750.

5. Diabetic Retinopathy Clinical Research Network, Elman MJ, Aiello LP, et al. Randomized trial evaluating ranibizumab plus prompt or deferred laser or triamcinolone plus prompt laser for diabetic macular edema. Ophthalmology 2010; 117:1064-1077.

6. Elman MJ, Ayala A, Bressler NM, et al. Intravitreal ranibizumab for diabetic macular edema with prompt versus deferred laser treatment: 5-year randomized trial results. Ophthalmology 2015; 122:375-381.

7. Brown DM, Schmidt-Erfurth U, Do DV, et al. Aflibercept for Diabetic Macular Edema: 100-Week Results from the VISTA and VIVID studies. Ophthalmology 2015; 122:2044-2052.

8. Mitchell P, Bandello F, Schmidt-Erfurth U, et al. The RESTORE study: ranibizumab monotherapy or combined with laser versus laser monotherapy for diabetic macular edema. Ophthalmology 2011; 118:615-625.

9. Pearce I, Banerjee S, Burton BJ, et al. Ranibizumab $0.5 \mathrm{mg}$ for Diabetic Macular Edema with Bimonthly Monitoring after a Phase of Initial Treatment: 18-Month, Multicenter, Phase IIIB RELIGHT Study. Ophthalmology 2015; 122:1811-1819.

10. Fung AE, Lalwani GA, Rosenfeld PJ, et al. An optical coherence tomography-guided, variable dosing regimen with intravitreal ranibizumab (Lucentis) for neovascular age-related macular degeneration. Am J Ophthalmol. 2007; 143:566-583.

11. Boyer DS, Heier JS, Brown DM, et al. A phase Illb study to evaluate the safety of ranibizumab in subjects with neovascular age-related macular degeneration. Ophthalmology 2009; 116:1731-1739. 
12. Holz FG, Amoaku W, Donate J, et al. Safety and efficacy of a flexible dosing regimen of ranibizumab in neovascular age related macular degeneration: the SUSTAIN study. Ophthalmology 2011; 118:663671.

13. Martin DF, Maguire MG, Fine SL, et al. Ranibizumab and bevacizumab for treatment of neovascular age-related macular degeneration: two-year results. Ophthalmology 2012; 119:1388-1398.

14. Busbee BG, Ho AC, Brown DM, et al. Twelve-month efficacy and safety of $0.5 \mathrm{mg}$ or $2.0 \mathrm{mg}$ ranibizumab in patients with sub foveal neovascular age-related macular degeneration. Ophthalmology 2013; 120:1046-1056.

15. Sugimoto $M$, Tsukitome $H$, Okamoto $F$, et al. Clinical preferences and trends of anti-vascular endothelial growth factor treatments for diabetic macular edema in Japan. J Diabetes Investig. 2019; 10:475-483.

16. Singer MA, Awh CC, Sadda S, et al. HORIZON: an open label extension trial of ranibizumab for choroidal neovascularization secondary to age-related macular degeneration. Ophthalmology 2012; 119:1175-1183.

17. Engelbert M, Zweifel SA, Freund KB. Long-term follow-up for type 1 (subretinal pigment epithelium) neovascularization using a modified "treat and extend" dosing regimen of intravitreal antivascular endothelial growth factor therapy. Retina 2010; 30:1368-1375.

18. Gupta OP, Shienbaum G, Patel AH, et al. A treat and extend regimen using ranibizumab for neovascular age-related macular degeneration clinical and economic impact. Ophthalmology 2010; 117:2134-2140.

19. Shienbaum G, Gupta OP, Fecarotta C, et al. Bevacizumab for neovascular age-related macular degeneration using a treatand-extend regimen: clinical and economic impact. Am J Ophthalmol. 2012; 153:468-473.

20. Oubraham H, Cohen SY, Samimi S, et al. Inject and extend dosing versus dosing as needed: a comparative retrospective study of ranibizumab in exudative age-related macular degeneration. Retina 2011; 31:26-30.

21. Toalster N, Russell M, Ng P. A 12-month prospective trial of inject and extend regimen for ranibizumab treatment of age-related macular degeneration. Retina 2013; 33:1351-1358.

22. Kim LN, Mehta H, Barthelmes D, et al. Meta-analysis of real-world outcomes of intravitreal ranibizumab for the treatment of neovascular age related macular degeneration. Retina 2016; 36:1418 -1431.

23. Prünte $C$, Fajnkuchen $F$, Mahmood S, et al. Ranibizumab $0.5 \mathrm{mg}$ treat-and-extend regimen for diabetic macular oedema: the RETAIN study. Br J Ophthalmol. 2015 pii: bjophthalmol2015-307249.

24. Sugimoto M, Ichio A, Nunome T and Kondo M. Two year result of intravitreal bevacizumab for diabetic macular edema using treat and extend protocol. Medicine (Baltimore). 2017; 96: e6406.

25. Payne JF, Wykoff CC, Clark WL, et al. Randomized Trial of Treat and Extend Ranibizumab With and Without Navigated Laser Versus Monthly Dosing for Diabetic Macular Edema: TREX-DME 2-Year Outcomes. Am J Ophthalmol. 2019; 202:91-99. 
26. Ebneter A, Waldmeier D, Zysset-Burri DC, et al. Comparison of two individualized treatment regimens with ranibizumab for diabetic macular edema. Graefes Arch Clin Exp Ophthalmol. 2017; 255:549555.

27. Diabetic Retinopathy Clinical Research Network, Wells JA, et al. Aflibercept, bevacizumab, or ranibizumab for diabetic macular edema. N Engl J Med. 2015; 372:1193-1203.

28. Mitchell P, McAllister I, Larsen M, et al. Evaluating the impact of intravitreal aflibercept on diabetic retinopathy progression in the VIVID-DME and VISTA-DME studies. Ophthalmology Retina 2018; 2: 988-996.

29. Writing Committee for the Diabetic Retinopathy Clinical Research N, Gross JG, Glassman AR, et al. Panretinal photocoagulation vs intravitreous ranibizumab for proliferative diabetic retinopathy: $A$ randomized clinical trial. JAMA. 2015; 314: 2137-2146.

30. Wykoff CC, Eichenbaum DA, Roth DB, Hill L, et al. Ranibizumab induces regression of diabetic retinopathy in most patients at high risk of progression to proliferative diabetic retinopathy. Ophthalmology Retina 2018; 2: 997-1009.

31. Sugimoto M, Ichio A, Mochida D, Tenma Y, Miyata R, Matsubara $H$, Kondo M. Multiple effects of intravitreal aflibercept on microvascular regression in eyes with diabetic macular edema. Ophthalmol. Retina 2019; 3:1067-1075.

32. Flynn HW, Smiddy WE. Diabetes and ocular disease, San Francisco: The Foundation of the American Academy of Ophthalmology 2000.

33. Ehlers JP, Wang K, Singh RP, et al. A Prospective Randomized Comparative Dosing Trial of Ranibizumab In Bevacizumab-Resistant Diabetic Macular Edema: The REACT Study. Ophthalmol Retina. 2018; 2: 217-224.

34. Freund KB, Korobelnik JF, Devenyi R, et al. Treat-and-extend regimens with anti-VEGF agents in retinal diseases: A literature review and consensus recommendations. Retina 2015; 35:1489-1506.

35. Julien S, Biesemeier A, Taubitz T, et al. Different effects of intravitreally injected ranibizumab and aflibercept on retinal and choroidal tissues of monkey eyes. $\mathrm{Br} J$ Ophthalmol. 2014; 98:813-825.

36. Niwa Y, Kakinoki M, Sawada T, et al. Ranibizumab and Aflibercept: Intraocular Pharmacokinetics and Their Effects on Aqueous VEGF Level in Vitrectomized and Nonvitrectomized Macaque Eyes. Invest Ophthalmol Vis Sci. 2015; 56:6501-6505.

37. Kanda A, Noda K, Saito W, et al. Aflibercept Traps Galectin-1, an Angiogenic Factor Associated with Diabetic Retinopathy. Sci Rep. 2015; 5:17946.

38. Schwarzer P, Ebneter A, Munk M, et al. One-Year Results of Using a Treat-and-Extend Regimen without a Loading Phase with Anti-VEGF Agents in Patients with Treatment-Naive Diabetic Macular Edema. Ophthalmologica. 2019; 241: 220-225

39. Diabetic Retinopathy Clinical Research Network, Browning DJ, Glassman AR, et al. Relationship between optical coherence tomography-measured central retinal thickness and visual acuity in diabetic macular edema. Ophthalmology 2007; 114: 525-536. 
40. Campochiaro PA, Brown DM, Pearson A, et al. FAME Study Group. Sustained delivery fluocinolone acetonide vitreous inserts provide benefit for at least 3 years in patients with diabetic macular edema. Ophthalmology 2012; 119:2125-2132.

41. Maturi RK, Bleau L, Saunders J, et al. A 12-month, single-masked, randomized controlled study of eyes with persistent diabetic macular edema after multiple anti-VEGF injections to assess the efficacy of the dexamethasone-delayed delivery system as an adjunct to bevacizumab compared with continued bevacizumab monotherapy. Retina 2015; 35:1604-1614.

42. Kaldırım H, Yazgan S, Atalay K, et al. Intravitreal dexamethasone implantation in patients with different morphological diabetic macular edema having insufficient response to ranibizumab. Retina 2018; 38:986-99.

\section{Tables}

Please see supplementary files section to access the tables.

\section{Figures}

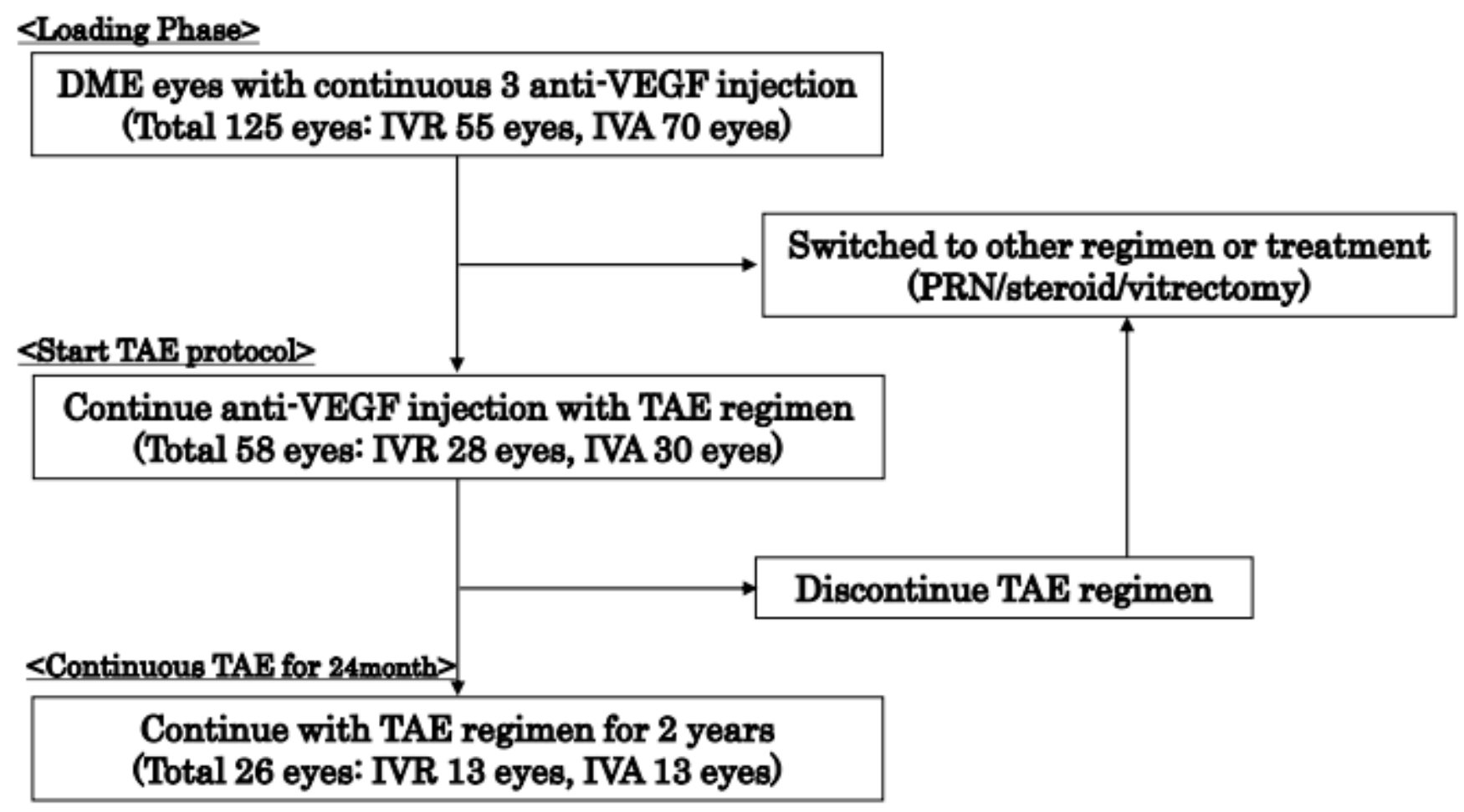

Figure 1

Flowchart of the study. A total 125 eyes from 125 patients (55 patients received IVR and 70 patients received IVA) diagnosed as treatment-naïve diabetic macular edema (DME) who had received 3consecutive monthly IVR or IVA injections were registered. Among them, 58 eyes of 58 patients (28 
patients received IVR and 30 patients received IVA) had injections with the TAE regimen during the 2 year maintenance period. In the end, 26 eyes of 26 patients (13 patients received IVR and 13 patients received IVA) completed the 2 year treatment with the TAE regimen.

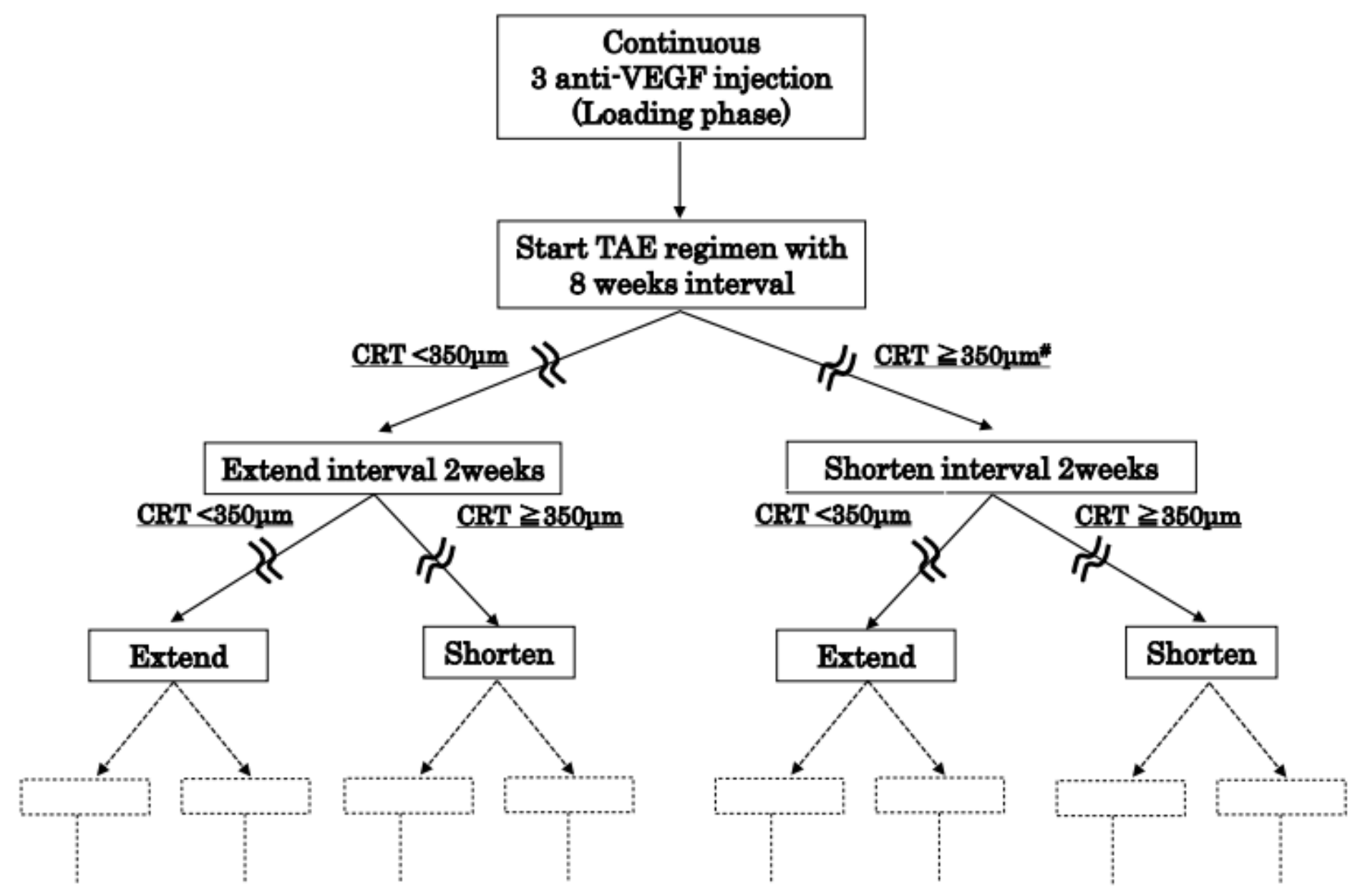

Figure 2

Modified-TAE regimen for diabetic macular edema (DME). All patients were given 3 consecutive monthly injections and they continued the IVR or IVA with a modified TAE regimen. The initial treatment interval after the loading phase was set at 8 weeks. The injection interval was extended by 2 weeks if the CRT was $<350 \mu \mathrm{m}$ at 2 consecutive examinations, and the injection interval was shortened by 2 weeks if the CRT was $\geq 350 \mu \mathrm{m}$. Black line, IVR; broken line, IVA. 


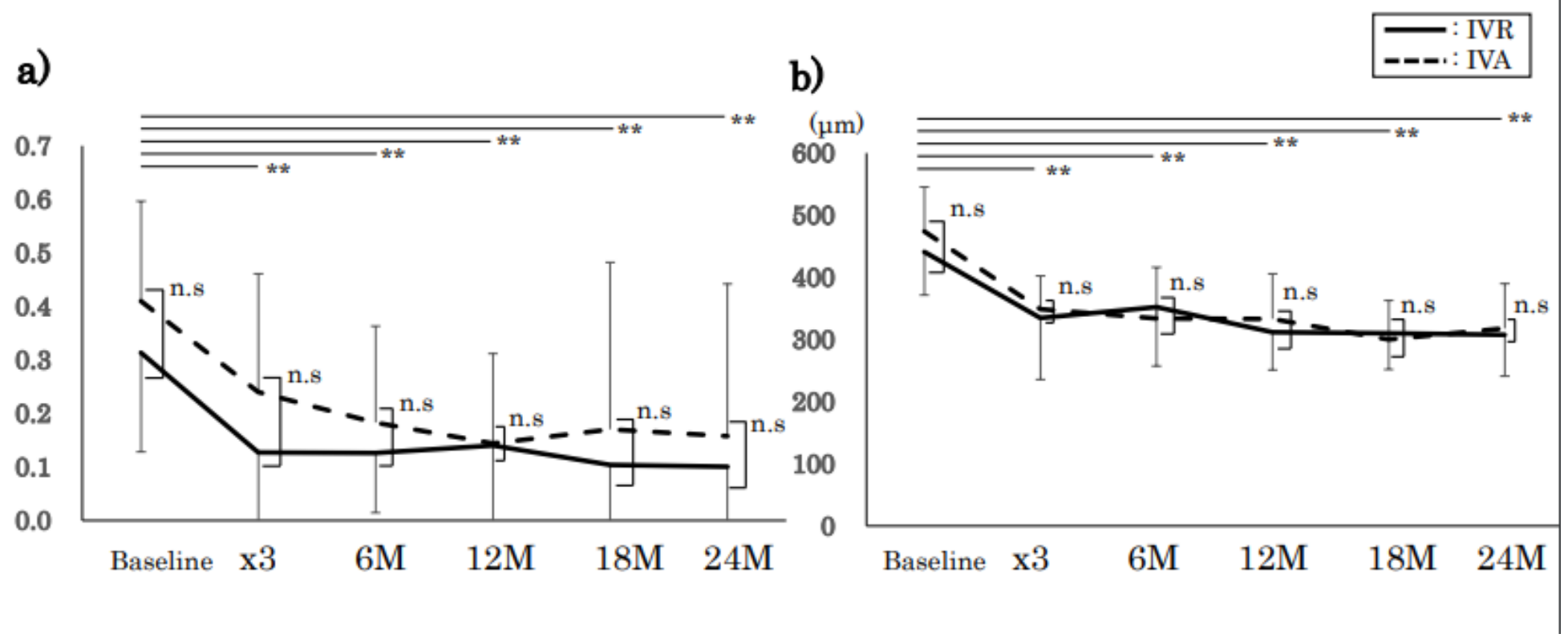

\section{Figure 3}

Changes in best-corrected visual acuity (BCVA) and central retinal thickness (CRT) following an intravitreal injection of ranibizumab (IVR) and aflibercept (IVA) applied with the Treat-and-Extend regimen. Two-way repeated measures ANOVA and post-hoc $t$ tests with Bonferroni's corrections were used to determine the significance of the changes in the BCVA and CRT. Kolmogorov-Smirnov tests were used to determine the significance of the differences between IVR and IVA. **: $P<0.01$. White circles, IVR; Black circles, IVA. 

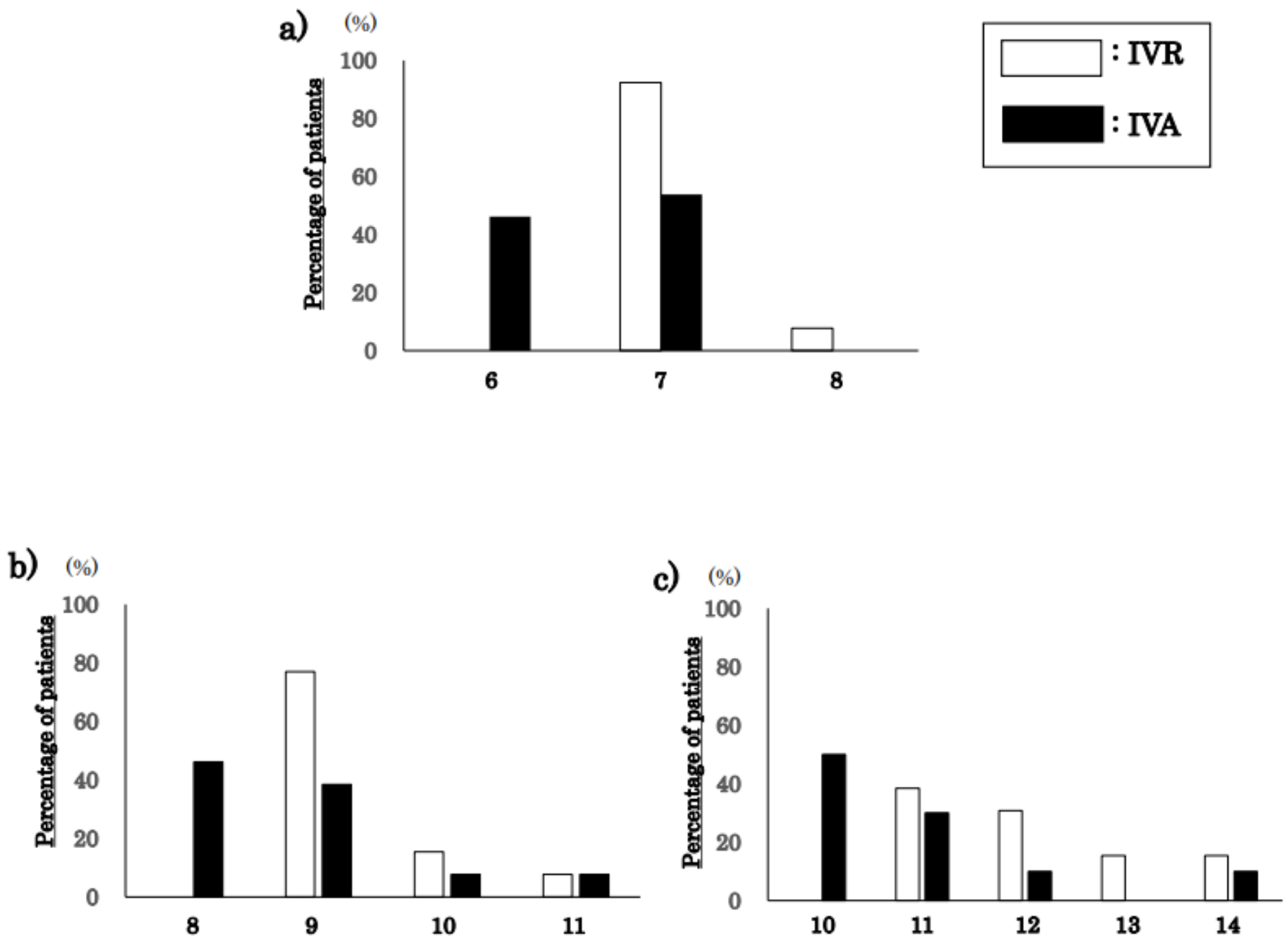

Figure 4

Number of intravitreal Injections of ranibizumab (IVR) and aflibercept (IVA). Number of injections for each observation period is shown. The bar graph shows the number of injections at 12 months (a), 18 months (b), and 24 months (c). Data are shown as the percentage of all patients.

\section{Supplementary Files}

This is a list of supplementary files associated with this preprint. Click to download.

- 20191228Chujyoetal.Tables.docx 